\title{
Fission in the landscape of heaviest elements: Some recent examples
}

\author{
J. Khuyagbaatar ${ }^{1,2, a}, A$. Yakushev², Ch.E. Düllmann ${ }^{1,2,3}$, D. Ackermann ${ }^{2, b}$, \\ L.-L. Andersson ${ }^{1}, M$. Block ${ }^{1,2,3}, H$. Brand $^{2}, J$. Even ${ }^{1,3, c}, U$. Forsberg $^{4}, W$. Hartmann $^{2}$, \\ R.-D. Herzberg ${ }^{5}$, F.P. Heßberger ${ }^{1,2}, J$. Hoffmann ${ }^{2}, A$. Hübner ${ }^{2}, E$. Jäger ${ }^{2}, J$. Jeppsson ${ }^{4}$,

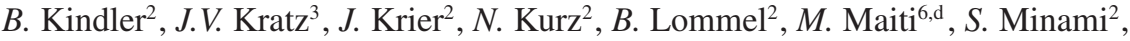 \\ D. Rudolph ${ }^{4}, J$. Runke $^{2}$, L.G. Sarmiento ${ }^{4}, M$. Schädel ${ }^{2}$, B. Schausten ${ }^{2}, J$. Steiner ${ }^{2}$, \\ $T$. Torres De Heidenreich ${ }^{2}, J$. Uusitalo ${ }^{7}, N$. Wiehl ${ }^{1,3}$, and $V$. Yakusheva ${ }^{2}$ \\ ${ }^{1}$ Helmholtz Institute Mainz, 55099 Mainz, Germany \\ ${ }^{2}$ GSI Helmholtzzentrum für Schwerionenforschung, 64291 Darmstadt, Germany \\ ${ }^{3}$ Johannes Gutenberg-Universität Mainz, 55099 Mainz, Germany \\ ${ }^{4}$ Lund University, 22100 Lund, Sweden \\ ${ }^{5}$ University of Liverpool, Liverpool L69 7ZE, UK \\ ${ }^{6}$ Saha Institute of Nuclear Physics, Kolkata 700064, India \\ ${ }^{7}$ University of Jyväskylä, 40351 Jyväskylä, Finland
}

\begin{abstract}
The fission process still remains a main factor that determines the stability of the atomic nucleus of heaviest elements. Fission half-lives vary over a wide range, $10^{-19}-10^{24} \mathrm{~s}$. Present experimental techniques for the synthesis of the superheavy elements that usually measure $\alpha$-decay chains are sensitive only in a limited range of half-lives, often $10^{-5}-10^{3} \mathrm{~s}$. In the past years, measurement techniques for very short-lived and very longlived nuclei were significantly improved at the gas-filled recoil separator TASCA at GSI Darmstadt. Recently, several experimental studies of fissionrelated phenomena have successfully been performed. In this paper, results on ${ }^{254-256} \mathrm{Rf}$ and ${ }^{266} \mathrm{Lr}$ are presented and corresponding factors for retarding the fission process are discussed.
\end{abstract}

\section{Introduction}

The stability, i.e. the existence of an atomic nucleus that consists of $Z$ protons and $N$ neutrons, strongly depends on the interplay between the attractive nuclear and repulsive Coulomb forces. In heavy nuclei the latter force eventually becomes dominant and spontaneous fission (SF) limits their stability [1]. Some observables of the fission process were successfully explained within the liquid drop model, which also predicted that nuclei with too many protons cannot exist due to the absence of a (macroscopic) fission barrier (e.g. $Z \gtrsim 112$ [2]). In the 1960's the shell-structure influence on the mass of the atomic nucleus has been investigated and accordingly the fission barrier was represented as a superposition of

\footnotetext{
${ }^{a}$ Corresponding author: J. Khuyagbaatar@gsi.de

${ }^{\mathrm{b}}$ Present address: GANIL, CEA/DSM-CNRS/IN2P3, Bd. Henri Becquerel, BP. 55027, 14076 Caen Cedex 5, France

${ }^{c}$ Present address: KVI-Center for Advanced Radiation Technology University of Groningen, 9747 AA Groningen, The Netherlands

${ }^{\mathrm{d}}$ Present address: Indian Institute of Technology Roorkee, Roorkee 247667, India
}

(C) The Authors, published by EDP Sciences. This is an Open Access article distributed under the terms of the Creative Commons Attribution License 4.0 (http://creativecommons.org/licenses/by/4.0/). 
the liquid-drop and shell-correction energy terms [3,4]. Within these earlier versions of the macro-microscopic models the next spherical magic numbers $Z=114$ and $N=184$ have been predicted and nuclei at around these numbers are inferred to be surprisingly stable against spontaneous fission. A possible next magic proton number has several times alternatively been allocated to $Z=120$ or 126 within modern self-consistent theoretical calculations $[5,6]$.

To date many superheavy nuclei (SHN) with $Z$ and $N$ up to 118 and 176, respectively, have been synthesized. The heaviest ones have been produced in ${ }^{48} \mathrm{Ca}$-induced fusion reactions with actinide targets and identified by their subsequent $\alpha$-decay chains, which always ended with fission of relatively neutron-rich unknown isotopes of elements down to $\mathrm{Db}$ [7].

One way for probing $Z$ and $A$ assignments of SHN is testing the systematics of observables like $\alpha$-decay energies and $\alpha$-decay $\left(T_{\alpha}\right)$ and SF $\left(T_{S F}\right)$ half-lives. Especially $T_{S F}$ of the relatively neutron-rich isotopes at the end of the $Z=114-118$ chains can be compared with systematics based on the well known neutron-deficient ones, where shell-structure effects are well established.

The shell structure of the nucleus can strongly affect the stability against fission, primarily on its mass surface. Such an influence can be studied using the even-even nuclei as it has been observed for $N=152$, where enhanced fission stabilities were found compared to neighbouring isotopes of $\operatorname{Fm}(Z=100)$ and No $(Z=102)$ elements. Single and multiparticle configurations also hinder SF, and the strength of the hindrance depends strongly on the corresponding nuclear orbitals, e.g. quantum numbers associated with intrinsic angular momentum.

Present experimental techniques for the detection and identification of SHN are limited: they are sensitive for fissioning nuclei with half-lives roughly between tens of $\mu$ s up to a few hours at most. The identification of nuclei with $T_{1 / 2}$ outside these limits is challenging at best using standard experimental data acquisition (DAQ) methods. DAQ systems based on fast sampling ADCs have been in use for about a decade for detection of charged particles [8]. Recently, such a method has successfully been implemented at the gas-filled recoil separator TASCA and has been used for the first time in experiments dedicated to syntheses of SHN [9-11]. Its advantages for the detection of $\alpha$ particles have already been described in Ref. [12]. In the present paper its benefits for the detection of SF-decaying nuclei will be demonstrated with examples of both short-lived $\left({ }^{254-256} \mathrm{Rf}[13]\right)$ and long-lived $\left({ }^{266} \mathrm{Lr}\right.$ [11]) isotopes. Each case corresponds to a particular effect of the shell structure on the fission stability. Accumulated data and analyses procedures of fission activities will also be presented: (i) Spontaneous fission of even-even ${ }^{254,256} \mathrm{Rf}$ will demonstrate the influence of the shell structure on the nuclear mass. (ii) An influence of the single-particle configuration on the fission stability will be shown with even-odd ${ }^{255} \mathrm{Rf}$. (iii) Fission hindrances due to multi-particle configurations will be demonstrated with even-even ${ }^{254} \mathrm{Rf}$ ( $K$-isomeric state) and odd-odd ${ }^{266} \mathrm{Lr}$.

\section{Experimental setup}

Since 2009 the gas-filled recoil separator TASCA is available for experiments aiming at SHN and has successfully been used for the studies of the elements flerovium ( $\mathrm{Fl}, Z=114$ ) [14-16]), moscovium (Mc, $Z=115$ ) [9], and Tennessine ${ }^{1}$ (Ts, $Z=117$ ) [11]. TASCA consists of a deflecting dipole and a focusing quadrupole doublet and can be operated in

\footnotetext{
${ }^{1}$ Here we adopt the suggested IUPAC names for elements 115 and 117.
} 
different ion-optical modes [17], and with various fill gases [18], depending on experimental requirements.

Nuclei considered in this work were produced as evaporation residues (ERs) of excited compound nuclei formed in nuclear fusion reactions between ${ }^{48} \mathrm{Ca}$ and ${ }^{50} \mathrm{Ti}$ projectiles (beam) and solid ${ }^{204,206,208} \mathrm{~Pb}[13]$ and ${ }^{249} \mathrm{Bk}[11,19]$ targets. Pulsed beams (pulse length: $5 \mathrm{~ms}$, repetition frequency: $50 \mathrm{~Hz}$ ) were accelerated by the UNIversal Linear ACcelerator (UNILAC) to energies around the Coulomb barrier. Typical target thicknesses were $0.5 \mathrm{mg} / \mathrm{cm}^{2}$. ERs leaving the target with relatively high charge states with broad distributions enter the gas-filled volume of TASCA, where charge-state equilibrating charge-exchange processes occur. After sufficiently many collisions the charge-state distribution becomes narrow at around an average one (often $q<10$ ), before reaching the dipole magnetic field. Consequently, the trajectory of the ERs through TASCA can be defined by adjusting the dipole magnetic field to their magnetic rigidity $[18,20]$. For the present studies TASCA was filled with helium gas at 0.8 mbar pressure. At the focal plane of TASCA, ERs were implanted into a double-sided silicon strip detector (DSSSD) comprising 144 vertical $(X)$ and 48 horizontal $(Y)$ strips on the front and back sides, respectively.

TASCA was significantly upgraded in 2011 by improving its performance including the assembling of a fast DAQ system for the implantation detectors [21]. All pre-amplifier signals from the $Y$-strips of the DSSSD were digitized by $60 \mathrm{MHz}$-sampling ADCs and stored in $50 \mu \mathrm{s}$-long traces. Signals from the $X$-strips of were processed with standard analog electronics and peak-sensing ADCs with a dead-time of $\leqslant 35 \mu$ s. These analog and digital branches, which independently initialized data storage, allowed determining the origin of events in two independent ways [11,12,21].

\section{Experimental results}

\subsection{SF of ${ }^{256} \mathrm{Rf}$, ${ }^{255} \mathrm{Rf}$ and ${ }^{254} \mathrm{Rf}$}

The isotope ${ }^{256} \mathrm{Rf}$ was produced in the ${ }^{50} \mathrm{Ti}\left({ }^{208} \mathrm{~Pb}, 2 n\right)$ reaction. A typical energy spectrum of fission fragments detected in the DSSSD by the analog part of the DAQ is shown in Fig. 1a. These high-energetic events were in anti-coincidence with signals from the MWPC. Spatial and time correlation analysis between them and preceding evaporation residues (hereafter noted as ER-SF) allowed to determine the half-life of ${ }^{256} \mathrm{Rf}$ [see Fig. 2a] according to a method described in Ref. [22]. Traces of these fission events were stored as saturated signals in the digital part of the data. The saturation is due to the limited dynamic input range of the sampling ADCs. The saturation time can be used as a variable for the determination of the energy deposited in the DSSSD by the fission fragments in the digital data, as shown in Fig. 1b. The time distribution of fission events from decay of even-odd ${ }^{255} \mathrm{Rf}$ produced via ${ }^{50} \mathrm{Ti}\left({ }^{206} \mathrm{~Pb}, 1 n\right)$ is shown in Fig. 2b. Due to the long half-life, 1.9(4) s, all fission traces were detected as single saturated signals similar to ${ }^{256} \mathrm{Rf}$.

${ }^{254} \mathrm{Rf}$ was produced in the ${ }^{50} \mathrm{Ti}\left({ }^{206} \mathrm{~Pb}, 2 n\right)$ reaction. Only a few events with saturated single signal, like the ones shown in Fig. 1a, were detected. They were found to be correlated with ERs within the short time as shown in Fig. 2c. The correlation time distribution of these events was terminated at about $50 \mu \mathrm{s}$, because most of the fission events from ${ }^{254} \mathrm{Rf}$ were recorded as a second signal in ER-traces. Thus, these fission events were lost in the analog part of the data as being an individual fission-like event! One of them is exemplified in Fig. 1d. Energies of the first signal in such traces were corresponding to ER energies similar to that observed from the decay of ${ }^{255,256} \mathrm{Rf}$. After extracting such short correlated ER-SF signals from the ER-traces, the full correlation time distribution was reconstructed; the corresponding halflife of ${ }^{254} \mathrm{Rf}$ was determined to $T_{S F}=20(3) \mu$ s. 

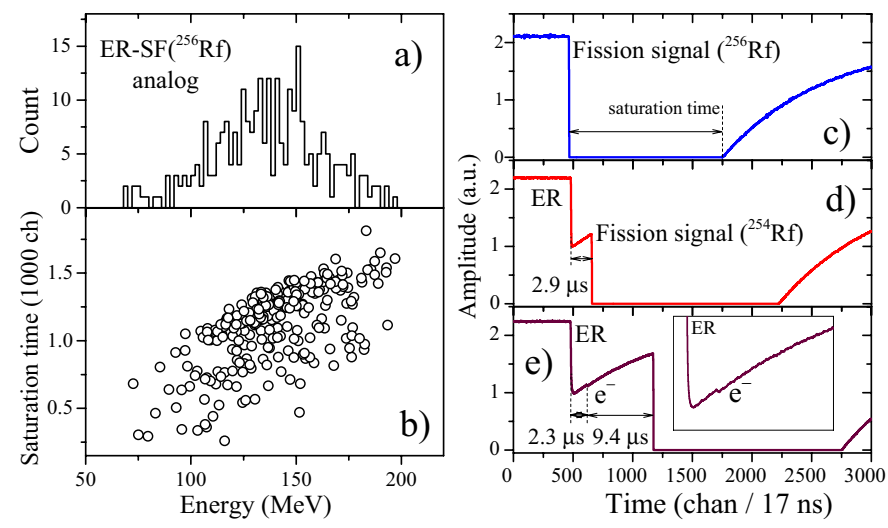

Figure 1. (a) Energy spectrum and (b) saturation times of traces versus energies of fission events from the decay of ${ }^{256} \mathrm{Rf}$ measured in the analog part of the data. (c)-(e) Examples of different types of traces containing fission events: (c) single-signal trace corresponding to ${ }^{256} \mathrm{Rf}$. (d) Double-signal trace where the implantation signal (noted by ER) of ${ }^{254} \mathrm{Rf}$ was followed by fission. (e) Triple-signal trace where ${ }^{254 m} \mathrm{Rf}$ was implanted and after $2.3 \mu$ s the isomeric state de-excited to the ground state which decays by SF after $9.4 \mu \mathrm{s}$. The insert highlights the region of the electron signal.

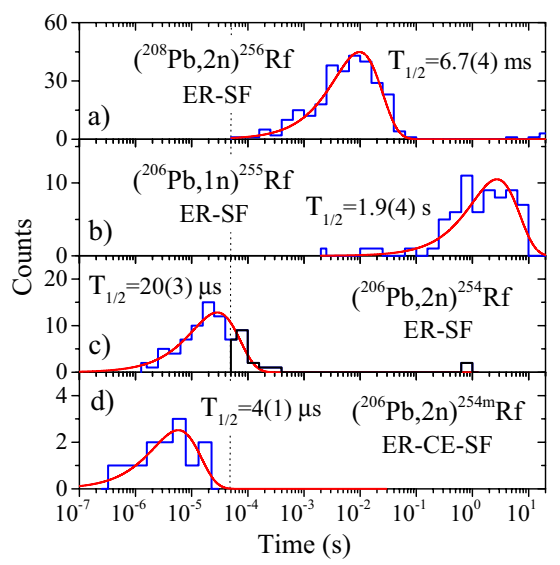

Figure 2. Correlation-time distribution of fission events relative to preceding ERs (a)-(c). The distribution of single-signal fission traces which terminated at $\approx 50 \mu$ s (marked by the dotted line) is also shown in panel (c). (d) Time distribution of electron-like signals relative to ERs correlated with fissions from ${ }^{254} \mathrm{Rf}$. Lines represent the calculated density distribution of the fission events on a logarithmic time scale according to Ref. [22]. The center of gravity of the distribution determines the lifetime ( $\left.\tau=1.443 \cdot T_{1 / 2}\right)$ of the fissioning nucleus. Half-lives of the corresponding isotopes with uncertainties (in the bracket) and reactions in which they were produced are given. See text for details.

Furthermore, in several traces of ER-like events a small signal was detected in between the ER and fission signals [23]. These signals with amplitudes not exceeding $500 \mathrm{keV}$ were attributed to originate from the registration of electrons. Time differences between the ER and these electron-like signals are shown in Fig. 1e. They represent an activity with a half-life of 4(1) $\mu$ s. Therefore, all observed events containing ER-electron-SF signals were attributed to implantation of ${ }^{254} \mathrm{Rf}$ in an isomeric state associated with the emission of conversion electrons (CEs). 


\subsection{Fission from ${ }^{266} \mathrm{Lr}$ and $\alpha$-decay assignment for ${ }^{270} \mathrm{Db}$}

The nucleus ${ }^{266} \mathrm{Lr}$ has not been produced directly so far. In 2012 at TASCA, this isotope was assigned to be produced in $\alpha$-decay chains associated with ${ }^{294} 117$ [11]. Its safe observation was possible thanks to several experimental conditions, e.g., the use of a highly pixelized DSSSD [15], fast electronics [11], the UNILAC pulsed beam, and well-tuned TASCA [20] that allowed to assign a long-lived $\alpha$ decay for the mother nucleus ${ }^{270} \mathrm{Db}$ in both $\alpha$-decay chains stemming from element 117 .

As an example, energies of events measured in the pixel $X=103$ and $Y=41$ [11] without a coincident signal from the MWPC are shown in Fig. 3 as a function of time counting from the beginning of the experiment. Events occurring in the DSSSD during beam off periods are marked by additional horizontal lines. Within the energy range of 7-12 MeV where most of the known $\alpha$ decays from heavy nuclei should occur, 55 beam-off events were detected. From the even-by-event analysis, 32 of them were identified as originating from known isotopes around ${ }^{208} \mathrm{~Pb}$ produced in transfer reactions (see Fig. 3).

Alpha decays in the region "northeast" of ${ }^{208} \mathrm{~Pb}$ occur with short half-lives. If the $\alpha$-particle energy is above $8 \mathrm{MeV}$ and it originates from nuclei with $Z \approx 82-90$ and $A \approx 210-230$ then the corresponding ERs can mostly be found in the data within $1 \mathrm{~s} \mathrm{[24].}$ Therefore, depending on the counting rate of the individual DSSSD pixel, the predominant amount of beam-off events can be identified.

Several events were detected within a short time window (cf. Fig. 3, cluster). They were unambiguously attributed to originate from element $Z=117$ based on the similarity to findings reported from the Dubna Gas-Filled Recoil Separator (DGFRS) [25-27]. Only one fission-like event was observed 5.1 hours after the last $\alpha$ from the cluster-event. In between, four $\alpha$-like events with energies of $6.82,7.89,11.53$ and $6.29 \mathrm{MeV}$ were measured. Only the $7.89-\mathrm{MeV}$ event could not be assigned to any known isotope. Furthermore, this was the only one event left without an assignment from in total seven events detected during the entire experiment within the energy range of 7.7-8.2 MeV, where a possible decay of ${ }^{270} \mathrm{Db}$ has been discussed in Ref. [27]. A similar situation has occurred in the second $\alpha$-decay chain, where the energy of the corresponding $\alpha$ event was $7.90 \mathrm{MeV}$ [11]. Therefore, these events were attributed to ${ }^{270} \mathrm{Db}$ and the fission events that terminate the decay chains to the decay of ${ }^{266} \operatorname{Lr}[11]$.

\section{Discussion and summary}

The influence of the shell structure on the spontaneous fission of heavy nuclei is well visible in systematics of $T_{S F}$, as shown in Fig. 4. The results on isotopes presented in this work $[11,18]$ are marked by solid symbols. Measured $T_{S F}$ of ${ }^{254-256} \mathrm{Rf}$ were in agreement with literature data [24]. Maxima of $T_{S F}$ of $\operatorname{Fm}(Z=100)$ and No $(Z=102)$ isotopes observed at $N=152$ show the enhanced fission stability due to the shell closure. However, even-even $\operatorname{Rf}(Z=104)$ isotopes do not anymore show a well pronounced peak at $N=152$ compared to lighter elements. This might be related to a weakened influence of the shell closure on the fission barrier. These nuclei are deformed, and thus the gaps between those nuclear orbitals responsible for the shell closure are strongly affected by interactions between the nucleons, i.e. both numbers of protons and neutrons. This is one of the distinct evidences of differences on the "magic" numbers ( $Z=100,108$ and $N=152,162)$ occurring in the deformed nuclei from those at, for example, $Z=82$ and $N=126$, where deformation is absent.

In case of nuclei with an unpaired proton and/or neutron (odd- $A$ Rf, $\mathrm{Lr}$ and $\mathrm{Db}$ ), $T_{S F}$ are significantly longer than in the neighbouring even-even ones, showing the effect of single and 


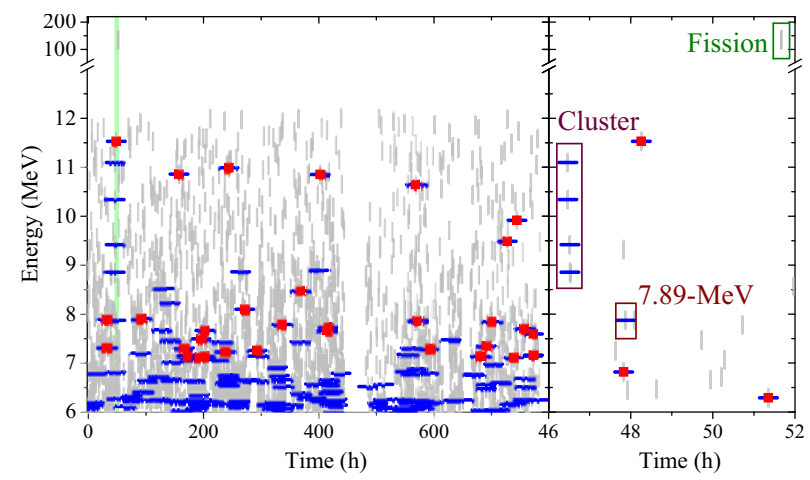

Figure 3. Energy versus time plot for events detected in pixel $X=103$ and $Y=41$ of the DSSSD where one of the $\alpha$-decay chains associated with ${ }^{294} 117$ was observed [11]. The left panel shows the full time period of the experiment. The right panel shows a detailed view of the period the chain was observed. Events without a coincident signal from the MWPC and occurring during beam-off periods are marked by horizontal and vertical lines, respectively. Events with energies in the range of 7-12 MeV (an additional two low-energy events in the right panel), which were identified to originate from the products of transfer reactions, are marked by additional solid squares.

multi-particle configurations on the fission process. We note that experimentally observed SF branches from odd- $A$ and/or odd-odd nuclei do not necessarily always correspond to the ground-state decay. Though $T_{S F}$ of odd- $A \mathrm{Rf}, \mathrm{Lr}$ and $\mathrm{Db}$ isotopes show a maximum at $N=153$ associated with the influence of both the $N=152$ shell closure and single and/or multi-particle configurations. An increase in $T_{S F}$ towards $N=162$ was observed in Rf-Hs, and in the case of $\mathrm{Db}$ a peak-like trend is observed at $N=163$ similar to the situation $N=153$, which indicates a shell closure.

An intriguing finding is the assignment of the long-lived $\alpha$ decay of ${ }^{270} \mathrm{Db}$. From the experimental data analysis' point of view, two identical cases of 7.9-MeV $\alpha$ 's were clearly found to be member of the decay chains. However, in Ref. [28], the non-observation of analogous $\alpha$-branching in the odd- $N$ neighbour ${ }^{268} \mathrm{Db}(N=163)$ was pointed out, questioning the assignment of $\alpha$ decay in ${ }^{270} \mathrm{Db}$. According to Ref. [28] $\alpha$-decay properties of ${ }^{268} \mathrm{Db}$ could be expected to be similar to those of ${ }^{270} \mathrm{Db}$. However, no $\alpha$ decay was identified and assigned for ${ }^{268} \mathrm{Db}$ in several experiments performed at DFGRS [28], TASCA [9] and Berkeley Gas-filled separator (BGS) [29]. However, we note that the DGFRS and BGS experiments were significantly less sensitive for measuring long-lived $\alpha$ decay because of the use of a DC beam where no (background-free) beam-off periods occur. The absence of an $\alpha$-decay branch in ${ }^{268} \mathrm{Db}$ would mean that it has a longer $T_{\alpha}$ compared to $\approx 1$ hour of the neighbouring ${ }^{270} \mathrm{Db}(N=165)$. Such cases are well known to occur in odd-odd and evenodd nuclei located above closed shells: for instance, $T_{\alpha}$ of ${ }^{256} \operatorname{Lr}(N=153)$ is about eight times longer than ${ }^{258} \operatorname{Lr}(N=155)$. In cases of spherically shaped nuclei such $T_{\alpha}$ differences between $N=127$ and 129 isotopes of odd- $Z$ elements vary up to $6 \cdot 10^{5}$. Thus, it would not be surprising to expect a longer $T_{\alpha}$ and different $\alpha$-decay energy for ${ }^{268} \mathrm{Db}$ than ${ }^{270} \mathrm{Db}$. For the future, more dedicated experiments to search for the long-lived $\alpha$ decay of ${ }^{268} \mathrm{Db}$ and more data on ${ }^{270} \mathrm{Db}$ need to be performed. Their results will help to shed light on the shell structure around $N=162$. We also note that in the case of odd-odd nuclei where both $\alpha$ decay and SF are hindered, other radioactive decay modes ( $\beta$ decay and electron capture) can become preferable. Recently, statistical analyses of the $\alpha$-decay chains attributed to start at $Z=115$ checking for the presence of such decays, have been carried out [31]. Their results show that 


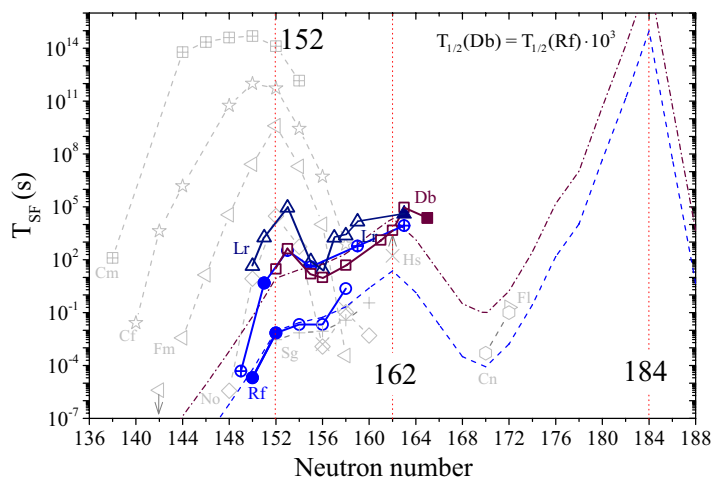

Figure 4. Partial SF half-lives of even-even isotopes of the heaviest elements including odd- $A$ Rf, Lr, and $\mathrm{Db}$ isotopes, as function of neutron number. Solid symbols mark experimental data provided in this paper, open symbols are from literature [24]. Theoretically calculated $T_{S F}$ of even-even $\mathrm{Rf}$ isotopes are also given as dashed lines [30]. Dashed-dotted lines represent the same calculation but multiplied by $10^{3}$, which simulates a hindrance factor due to an unpaired proton and/or neutron. Location of known and predicted neutron shell closures are marked by vertical dotted lines.

different scenarios for the observed $\alpha$-decay chains can be proposed, assuming that one or more of the chain-members underwent electron capture decay.

Apparently, the identification of long-lived nuclei around $N=162$ (expected also at $N=184$ ) turned out to be a major task for further SHN studies. Suitable experimental techniques have to be implemented.

Similarly, very short-lived fissioning nuclei can also be studied in the future as demonstrated in the case of ${ }^{254} \mathrm{Rf}$ and its isomeric state. The latter observation made through electron detection without requesting a particular trigger was possible thanks to a fast DAQ, whose further advantage for the solution of fission related topics still needs to be explored. For instance, the observed ER-CE-SF sequence for ${ }^{254} \mathrm{Rf}$ indicates that an isomeric state is de-exciting into the ground state instead of direct fission. When such situation occurs in the strongly spontaneously fissioning nucleus then the most probable origin of the isomer is an excited state with high $K$-quantum number. $K$ represents the projection of the total nuclear spin along the symmetry axis of the nucleus [32, 33]. The same isomeric state in ${ }^{254} \mathrm{Rf}$ has recently been identified and half-lives are in agreement [34]. A two-quasi-neutron configuration $\nu 7 / 2^{+}[624] \otimes \nu 9 / 2^{-}[734]$ forming $K=8$ has been suggested for this $4-\mu \mathrm{s}$ isomeric state in Ref. [34]. An additional 247- $\mu$ s isomeric state has also been observed. In the present experiment a threshold for the detection of signals in the DSSSD were not sensitive enough for measurements of electrons, thus the $247-\mu$ s-isomeric decays reported in Ref. [34] could not be observed.

$K$-hindered fission stability might become an important issue for further studies of ultra short-lived nuclei with extreme numbers of $Z$ and $N$. Very neutron-deficient nuclei with $T_{S F}$ much shorter than $1 \mu \mathrm{s}$, which is approximately the flight-time of ERs through short in-flight separators for SHN, are just one example. Such an experimental scenario has been applied at TASCA for the hitherto unknown ${ }^{252} \mathrm{Rf}$ with a predicted $T_{S F}=0.6 \mu$ s [30]; these results will be presented elsewhere [23].

We are grateful for GSI's ECR ion-source and UNILAC staff, and the Experimental Electronics department for their continuous support of the experiments. 


\section{References}

[1] R. Vandenbosch and J.R. Huizenga, Nuclear Fission (Academic Press, New York, 1973).

[2] A. Sobiczewski and I. Muntian, Nucl. Phys. A734, 176 (2004).

[3] A. Sobiczewski et al., Phys. Lett. 22, 500 (1966).

[4] W.D. Myers and W.J. Swiatecki, Nucl. Phys. 81, 1 (1966).

[5] K.M. Rutz et al., Phys. Rev. C 56, 238 (1997).

[6] S. Čwiok et al., Nucl. Phys. A611, 211 (1996).

[7] J.H. Hamilton, S. Hofmann, Yu.Ts. Oganessian, Ann. Rev. Nucl. Part. Sc. 63, 383 (2013).

[8] S.N. Liddic et al., Phys. Rev. Lett. 97, 082501 (2006).

[9] D. Rudolph et al., Phys. Rev. Lett. 111, 112502 (2013).

[10] J. Khuyagbaatar et al., GSI Scientific Report-2012, 131 (2013).

[11] J. Khuyagbaatar et al., Phys. Rev. Lett. 112, 172501 (2014).

[12] J. Khuyagbaatar et al., Phys. Rev. Lett. 115, 242502 (2015).

[13] J. Khuyagbaatar et al., GSI Scientific Report-2012, 133 (2013).

[14] Ch.E. Düllmann et al., Phys. Rev. Lett. 104, 252701 (2010).

[15] J.M. Gates et al., Phys. Rev. C 83, 054618 (2011).

[16] A. Yakushev et al., Inorg. Chem. 53, 1624 (2014).

[17] A. Semchenkov et al., Nucl. Instr. Meth. B266, 4153 (2008).

[18] J. Khuyagbaatar et al., Nucl. Instr. Meth. A689, 40 (2012).

[19] J. Runke et al., J. Radioanal. Nucl. Chem. 299, 1081 (2014).

[20] J. Khuyagbaatar et al., Phys. Rev. A. 88, 042703 (2013).

[21] A. Yakushev et al., to be published.

[22] K.H. Schmidt et al., Z. Phys. A316, 19 (1984).

[23] J. Khuyagbaatar et al., to be published.

[24] http://www.nndc.bnl.gov/ensdf/.

[25] Yu.Ts. Oganessian et al., Phys. Rev. Lett. 104, 142502 (2010).

[26] Yu.Ts. Oganessian et al., Phys. Rev. Lett. 109, 162501 (2012).

[27] Yu.Ts. Oganessian et al., Phys. Rev. C. 87, 054621 (2013).

[28] Yu.Ts. Oganessian and V.K. Utyonkov, Rep. Prog. Phys. 78, 036301 (2015).

[29] J.M. Gates et al., Phys. Rev. C 92, 021301(R) (2015).

[30] R. Smolanczuk, J. Skalski, A. Sobiczewski, Phys. Rev. C 52, 1871 (1995).

[31] U. Forsberg et al., Nucl. Phys. A953, 117 (2016).

[32] D. Peterson et al., Phys. Rev. C 74, 014316 (2006).

[33] H.B. Jeppesen et al., Phys. Rev. C 79, 031303 (2009).

[34] H.M. David et al., Phys. Rev. Lett. 115, 132502 (2015). 